\title{
Pickering Emulsions Stabilized by Cellulose
}

\section{Nanocrystals Grafted with Thermo-responsive Polymer}

\section{Brushes}

\author{
Justin O. Zoppe, ${ }^{l}$ Richard A. Venditti, ${ }^{1}$ and Orlando J. Rojas ${ }^{1,2, *}$ \\ ${ }^{1}$ Department of Forest Biomaterials, North Carolina State University, Campus Box 8005, Raleigh, NC \\ 27697-8005, USA \\ ${ }^{2}$ Faculty of Chemistry and Materials Sciences, Department of Forest Products Technology, Aalto \\ University, P.O. Box 16300, FI-00076, Aalto, Finland
}

*Corresponding author: E-mail: ojrojas@ncsu.edu, Phone: +1-919-513 7494, Fax: +1-919-515 6302

\begin{abstract}
Cellulose nanocrystals (CNCs) from ramie fibers are studied as stabilizers of oil-in-water emulsions. The phase behavior of heptane and water systems are studied and emulsions stabilized by CNCs are analyzed by using drop sizing (light scattering) and optical, scanning and freeze-fracture electron microscopies. Water-continuous Pickering emulsions are produced with cellulose nanocrystals $(0.05$ to 0.5 wt \%) grafted with thermo-responsive poly(NIPAM) brushes (poly(NIPAM)-g-CNCs). They are observed to be stable during the time of observation of four months. In contrast, unmodified CNCs are unable to stabilize heptane-in-water emulsions. After emulsification poly(NIPAM)-g-CNCs are observed to form aligned, layered structures at the oil-water interface. The emulsions stabilized by
\end{abstract}


poly(NIPAM)-g-CNCs break after heating at a temperature above the LCST of poly(NIPAM), which is taken as indication of the temperature-responsiveness of the brushes installed in the particles and thus the responsiveness of the Pickering emulsions. This phenomenon is further elucidated via rheological measurements, in which viscosities of the Pickering emulsions increase on approach of the low critical solution temperature of poly(NIPAM). The effect of temperature can be counterbalanced with the addition of salt which is explained by the reduction of electrostatic and steric interactions of poly(NIPAM)-g-CNCs at the oil-water interface.

KEYWORDS. Pickering emulsions, oil-in-water emulsions, Cellulose nanoparticles; cellulose nanocrystals, thermo-responsive emulsions, poly(N-isopropylacrylamide), LCST, grafts, SurfaceInitiated Single-Electron Transfer Living Radical Polymerization.

\section{INTRODUCTION}

The irreversible adsorption of solid particles at the oil-water interface has been known for over a century [1]. Such phenomenon is critical in the stabilization of surfactant-free emulsions, in the socalled Pickering emulsions [2], which have recently found use in applications spanning cosmetics, biomedical, and food products [3]. The high stability of emulsions stabilized by colloidal particles is derived from the energy barrier required to remove the particles from the interface in order to facilitate droplet coalesce. Pickering emulsions have a number of advantages over conventional surfactantstabilized emulsions in that they can reduce tissue irritation and their viscosity can be easily adjusted by solid content and/or solid type [4]. Nanoparticles modified with surface-active polymers have been shown to be very efficient emulsifiers with nanoparticle concentrations as low as 0.04 wt \% [5]. In addition, the colloidal assembly of solid particles within Pickering emulsions can be used as templates for advanced materials such as Janus colloids [6], composite microcapsules [7-9] or microspheres [10]. In particular, recent efforts focused on the development of environmentally friendly systems have led to bio-based materials such as renewable nanocomposite foams [11] and bio-inorganic microcapsules [12] from Pickering emulsion templates. 
The development of renewable biomaterials for advanced applications has been recently gained momentum in the research community $[13,14]$. Cellulose, in particular, is an attractive material source due to its availability, biodegradability, renewability, and amenability for surface modification. Cellulose fibers, containing crystalline and amorphous regions, can be subjected to strong acid hydrolysis yielding rod-like crystals having even more unique surface, optical and mechanical properties $[15,16]$. Cellulose nanocrystals (CNCs) or nanocrystalline cellulose (NCC) produced by sulfuric acid hydrolysis yields aqueous suspensions electrostatically-stabilized by sulfate ester groups installed on the surface which promotes uniform aqueous dispersions [17], and the ability to selfassemble into chiral nematic liquid crystals [18]. The utility of nanocellulosic nanoparticles as stabilizer of Pickering emulsion is expected to be advantageous over some inorganic nanoparticles when biocompatibility, degradability, density and cost issues are considered.

Due the hydrophilicity of cellulose surfaces, surface chemical modification is needed in order to impart an appropriate balance of surface energies or amphiphilicity, especially when stabilizing waterin-oil (w/o) emulsions [19]. In the past, microcrystalline cellulose (MCC) has been shown to stabilize oil-in-water (o/w) emulsions through network formation around oil droplets without surface modification [20, 21]. The long-term stabilization of Pickering emulsions and foams with microparticles from hydrophobic cellulose has been shown by Wege, et al. [22] Ougiya et al. first studied the use of unmodified bacterial cellulose to stabilize o/w emulsions in 1997 [23]. In addition, a number of patents have been filed involving the use of fibrillated cellulose as emulsion stabilizers [24, 25]. Furthermore, recent efforts have focused on the preparation of w/o emulsions by using silylated micro- or nanofibrils from wood pulp and bacterial cellulose [11, 26-29]. The utility of bacterial cellulose nanocrystals as a Pickering emulsion stabilizer has been recently demonstrated by Kalashnikova et al. [30]

As previously noted, Pickering emulsifiers irreversibly adsorb at the oil-water interface and require a much higher energy for desorption as compared to conventional surfactants. The use of responsive polymer grafts that react to changes in their environment, such as light, heat, ionic strength, and $\mathrm{pH}$ [3133] can be used as a means to control Pickering emulsion stability [34]. Thermo-responsive polymers 
may have an upper or a lower critical solution temperature (UCST and LCST), depending on entropic changes and phase behaviors associated with their molecular structure. A number of researchers have demonstrated thermo-responsive Pickering emulsions mostly by the use of (2-(dimethylamino)ethyl methacrylate)-carrying silica and polystyrene latex nanoparticles [34, 35]. Poly(N-isopropylacrylamide) (poly(NIPAM)), one of the most studied thermo-responsive polymers, has an LCST in aqueous solution ranging between 30 to $35{ }^{\circ} \mathrm{C}$ [36]. Above the LCST poly(NIPAM) phase-separates due to thermaldriven chain dehydration. Since the LCST of poly(NIPAM) is near the physiological temperature of ca. $37^{\circ} \mathrm{C}$, it has been extensively used in applications involving controlled drug release [37]. In addition, poly(NIPAM) has been shown to be surface-active due to the presence of amide and isopropyl functional groups [38-40]. The surface activity of particles containing poly(NIPAM) functionalities have been confirmed at the air-water interface [41, 42]. Pickering emulsions stabilized by particles carrying copolymers of NIPAM, methacrylic acid [43, 44], and acrylic acid [45] have been demonstrated. Most recently, silica [46] and polystyrene [47] particles modified by poly(NIPAM) have been developed as thermo-responsive Pickering emulsion stabilizers.

We have reported on the synthesis of poly(NIPAM) brushes grafted from CNCs via surface-initiated single-electron transfer living radical polymerization (SI-SET-LRP) [48] and their surface interaction forces [49]. The thermo-responsive behavior of poly(NIPAM)-g-CNCs and response to ionic strength was demonstrated. The aim of this study was to demonstrate the use of cellulose nanocrystals grafted with thermo-responsive functionalities as stabilizers of $\mathrm{o} / \mathrm{w}$ Pickering emulsions. The effect of solids content, temperature and ionic strength on emulsion stability and droplet size was investigated. The self-assembly of poly(NIPAM)-g-CNCs at the oil-water interface was visualized via Freeze-Fracture Electron Microscopy (FFEM) and Scanning Electron Microscopy (SEM).

\section{EXPERIMENTAL}

Materials. Pure ramie fibers were obtained from Stucken Melchers GmbH \& Co. (Germany). 2bromoisobutyryl bromide (BriB), 2-dimethylaminopyridine (DMAP), tetrahydrofuran (THF, 99\%), Nisopropylacryalamide (NIPAM), copper (I) bromide and $N, N, N^{\prime}, N^{\prime \prime}, N^{\prime \prime}$-pentamethyldiethylenetriamine 
(PMDETA), were all obtained from Sigma-Aldrich. Sulfuric acid (95\%), acetic acid (glacial), triethylamine (TEA, 99.5\%), acetone (99\%), ethanol (95\%), methanol (99\%), heptane (99\%), sodium hydroxide pellets, and sodium chlorite were all purchased from Fisher Scientific. All solvents were dried over molecular sieves ( $3 \AA$, 4-8 mesh beads, Sigma-Aldrich) for $48 \mathrm{~h}$ before use.

Cellulose nanocrystals and surface functionalization. Poly(NIPAM) chains were grafted from cellulose nanocrystals (CNCs) by Surface-Initiated Single-Electron Transfer Living Radical Polymerization (SI-SET-LRP). Details about this procedure and main properties of the obtained poly(NIPAM)-g-CNCs can be found in our earlier reports [48, 49]. The surface charge of unmodified ramie $\mathrm{CNCs}$ was determined to be $0.30 \mathrm{e} / \mathrm{nm}^{2}$ by conductometric titration. The CNCs utilized in this study were carrying poly(NIPAM) grafts polymerized from their surfaces with a molar ratio between initiator $[\mathrm{Br}]$ and anhydroglucose units $[\mathrm{AGU}]$ in $\mathrm{CNCs}([\mathrm{Br}]:[\mathrm{AGU}])$ of $5: 3$ and a number average molecular weight $\left(M_{n}\right)$ of $12,170 \mathrm{~g} / \mathrm{mol}$.

Water Contact Angle. The initial water contact angle (WCA) of spin-coated films of both unmodified and poly(NIPAM) grafted CNCs (poly(NIPAM)-g-CNCs) [49] were determined using a Phoenix 300 contact angle analyzer (SEO Co. Ltd, Lathes, South Korea). Contact angles were measured at room temperature and immediately after being placed in an oven at $80^{\circ} \mathrm{C}$ for 1 hour.

Preparation of Pickering Emulsions. All emulsions (total volume $10 \mathrm{~mL}$ ) were prepared with a water-to-oil ratio (WOR) of one containing given concentrations of poly(NIPAM)-g-CNCs in the aqueous phase and emulsified with an Ultraturrax T18 basic homogenizer (IKA, Wilmington, NC USA) at $6,000 \mathrm{rpm}$ for 60 seconds at $25^{\circ} \mathrm{C}$. Volume fractions of the organic and aqueous phases were noted 30 minutes and 4 days after emulsification at $25{ }^{\circ} \mathrm{C}$. The stability index of Pickering emulsions was determined by the change in volume fraction of emulsified phase from 30 minutes after preparation to 4 days. The type of emulsion was determined by the drop test. A drop of emulsion was added to neat water and neat heptane and their dispersion ability was observed. An oil-in-water emulsion disperses readily in water, while a water-in-oil emulsion disperses readily in heptane (respective continuous phases). 
Optical Microscopy. Emulsion droplets were visualized using an Olympus BH2-UMA optical microscope (Olympus, Center Valley, Pennsylvania, USA). Emulsion droplets were placed directly onto a glass microscope slide and viewed under 5-50x magnification.

Particle Size Analysis. Particle size distribution of emulsion droplets were obtained using a Horiba LA-910 Laser Scattering Particle Size Distribution Analyzer (Horiba Instruments, Inc., Irvine, CA USA) using the refractive index of heptane (1.39) [50]. The instrument reservoir was filled with water, and $1 \mathrm{~mL}$ of emulsion phase was added and circulated during the course of the measurement. The particle size distribution was calculated on a number average basis.

Rheology. Viscosity measurements of unmodified $\mathrm{CNCs}$ and Pickering emulsions with poly(NIPAM)-g-CNCs were obtained by using a TA Instruments AR 2000 Rheometer. A Peltier plate and $25 \mathrm{~mm} 1.59^{\circ}$ cone geometry was used to obtain viscosity at a temperature ramp range of 25 to 45 ${ }^{\circ} \mathrm{C}$ at constant shear rate of $100 \mathrm{~s}^{-1}$.

Freeze-fracture Electron Microscopy. Emulsion samples were placed in a gold freeze-fracture carrier (Technotrade International, Manchester, NH USA) and frozen by placing into re-solidifying propane cooled with liquid nitrogen. After knife fracture in the freeze-fracture instrument (CFE-50; Cressington, Watford, UK), emulsion samples were subjected to etching to remove ice with a liquidnitrogen-cooled knife and raising the stage temperature. Shadows were deposited with an electron gun (2-3 nm Pt/C; $\left.45^{\circ}\right)$ and replicated $\left(6-8 \mathrm{~nm}\right.$ carbon; $\left.90^{\circ}\right)$. Freeze-fractured samples were deposited on Formvar-coated copper mesh grids and observed with a Hitachi HF-2000 transmission electron microscope (TEM) operated at an accelerating voltage of $80 \mathrm{kV}$.

Scanning Electron Microscopy. A Hitachi S-3200N variable pressure scanning electron microscope (SEM) was used to image freeze-fractured emulsion samples. The samples deposited on Formvarcoated copper mesh grids were directly observed under a microscope working distance between 3 and $60 \mathrm{~mm}$ using an accelerating voltage from 0.3 to $30 \mathrm{kV}$.

\section{RESULTS AND DISCUSSION}


Cellulose nanocrystals from ramie fibers were produced and characterized according to a procedure reported previously [48]. The size of the obtained rod-like CNCs was determined to be 3-15 nm in diameter and 50-250 nm in length. Poly(N-isopropylacrylamide) was grafted from CNCs via surfaceinitiated single-electron transfer living radical polymerization (SI-SET-LRP) with various reaction conditions producing a variety of graft densities and molecular weights. Of the various molecular weights of the polymer grafts obtained, one case was examined as Pickering emulsion stabilizer on the basis of work by Saigal et al. [34] in which silica nanoparticles with a relatively low graft density of (2(dimethylamino)ethyl methacrylate) (PDMAEMA) was shown to be the most robust and efficient emulsifier. One of the reasons for the high emulsifying efficiency of low graft density nanoparticles includes their high-affinity adsorption isotherm at the oil-water interface which enables use at low particle concentrations. The CNCs utilized in this study were carrying poly(NIPAM) grafts polymerized from their surfaces with a molar ratio between initiator [Br] and anhydroglucose units [AGU] in CNCs ([Br]:[AGU]) of 5:3 and a number average molecular weight $\left(M_{n}\right)$ of $12,170 \mathrm{~g} / \mathrm{mol}$. The degree of surface substitution (DSS) of polymer brushes could not be determined by previous methods due to limited detection of bromine-containing initiator and the likely interference from adsorbed contaminants. Thus, the density of poly(NIPAM) grafts were inferred from the molecular weight and reaction conditions [48].

Pickering emulsions were prepared by first sonicating in water a given amount of poly(NIPAM)-gCNCs, followed by mixing with an equivalent amount of the organic phase (heptane). In the lowest concentration case $(0.05 \%)$, the effect of ionic strength of the aqueous media was also investigated. Emulsions prepared with unmodified CNCs were used as control. After homogenization for one minute at 6,000 rpm, volume fractions of emulsions were measured for stability after 30 minutes and 4 days and were observed during a period of about four months.

To verify the wettability of unmodified CNCs and poly(NIPAM)-g-CNCs with water and the heptane, contact angle measurements were performed on spin-coated films of the nanoparticles [49]. As shown in Table 1, the water contact angle (WCA) of unmodified CNCs was $22^{\circ}$ at ambient conditions ( $\mathrm{T}<\mathrm{LCST})$. After being placed in an oven the contact angle of CNCs at $40{ }^{\circ} \mathrm{C}(\mathrm{T}>\mathrm{LCST})$, decreased 
slightly as expected due to the decreased surface tension of water at the higher temperature. However, in the case of poly(NIPAM)-g-CNCs the opposite effect with temperature was noted: Initially, below the LCST of poly(NIPAM), the WCA was determined to be $38^{\circ}$, however, after being heated above the LCST, the WCA increased to $44^{\circ}$. This was expected due to the insolubility of poly(NIPAM) in water above the LCST, which led to a decreased wettability of the spin coated film, thus a slightly less hydrophilic surface. Although the increase in water contact angle is minimal given the error of the measurement, it is relevant here to note the opposed effect compared to that of unmodified CNCs. Additionally, the heptane contact angle (HCA) was measured to determine nanoparticle wettability with the oil phase of subsequent Pickering emulsions. Due to the low surface tension of heptane, spin coated films of unmodified and grafted CNCs showed a low HCA, ca. 14-16 ${ }^{\circ}$ The slightly lower HCA of poly(NIPAM)-g-CNCs was explained by the increased wettability with the organic phase of poly(NIPAM) grafts due to the presence of isopropyl groups within polymer chains.

Table 1. Initial water contact angle (WCA) and heptane contact angle (HCA) of spin-coated films of unmodified and grafted CNCs with temperature.

\begin{tabular}{lccc}
\hline Sample & WCA $\left(\mathbf{T}<\mathbf{L C S T}^{\circ} \mathbf{C}\right)$ & WCA $\left._{(\mathbf{T}}>\mathbf{L C S T}^{\circ} \mathbf{C}\right)$ & HCA $\left._{(\mathbf{T}}=\mathbf{2 5}^{\circ} \mathbf{C}\right)$ \\
\hline Unmodified CNCs & 22 & 18 & 16 \\
Poly(NIPAM)-g-CNCs & 38 & 44 & 14 \\
\hline
\end{tabular}

It is well known that Pickering emulsion stability depend on a number of parameters, such as particle concentration, particle shape, surface activity and ionic strength $[3,34]$. The concentration of nanoparticles utilized will determine the droplet size formed and the stability of the emulsion droplets to coalescence. Ionic strength plays an important role in regulating the electrostatic interactions that may occur between adjacent nanoparticles at the oil-water interface. In the case of nanoparticles grafted with thermo-responsive polymers, such as poly(NIPAM), ionic strength is also critical in the solvation of polymer brushes in aqueous media. Therefore, besides temperature, nanoparticle concentration and ionic strength of the aqueous phase were chosen as variables to study the stability of Pickering 
emulsions stabilized by poly(NIPAM)-g-CNCs. In addition, attempts were made to use unmodified CNCs as Pickering emulsion stabilizers as a control. Emulsions prepared with equal amounts of heptane and water (water-to-oil ratio of $1, \mathrm{WOR}=1$ ) after mixing without stabilizer, with unmodified CNCs, and with poly(NIPAM)-g-CNCs indicated that the two phases separated if no stabilizer was added or if unmodified CNCs were used (concentrations up to $2 \%$ CNCs were tested with no success). These results were contradictory to the findings of Kalashnikova et al. [30], in the case of bacterial CNC. In the aforementioned study, bacterial CNCs were produced via hydrochloric acid hydrolysis which left CNCs with very low charge density. In the present study, CNCs were produced from sulfuric acid hydrolysis which introduced negatively charged sulfate groups on the surface $\left(0.30 \mathrm{e} / \mathrm{nm}^{2}\right)$ which was expected to hinder emulsion stability due to electrostatic repulsion between adjacent nanoparticles at the oil-water interface. Additionally, the inability of unmodified CNCs to be dispersed in non-polar solvents is an indication of their primarily hydrophilic character $[15,16]$. In studies of ellipsoidal colloids at interfaces, it has been shown that the maximum packing of particles depends highly on the aspect ratio [3], which is an important parameter governing Pickering emulsions stability. Since, unmodified CNCs and grafted CNCs were expected to have similar aspect ratio, the presence of poly(NIPAM) brushes was proposed to play the primary role in their ability to tightly pack at the oilwater interface. This was proposed to be the result of the screening of electrostatic repulsion between adjacent nanoparticles and the surface activity imparted by poly(NIPAM) [38-40]. Interestingly, when poly(NIPAM)-g-CNCs was employed at low concentrations (for example, $0.05 \%$ oil-in-water emulsions were formed and were noted to be stable for at least the time of observation of four months.

Effect of nanoparticle concentration. Table 2 shows the compositions and stability indices calculated for heptane-in-water emulsions containing poly(NIPAM)-g-CNCs $(0.05$ to $0.5 \mathrm{wt} \%$ in the aqueous phase) and unmodified CNCs. As noted before, equivalent concentrations of unmodified CNCs were not able to stabilize the emulsions with heptane and oil (extreme, very high concentrations of CNCs were required in order to produce a small fraction of an emulsified phase, $2.2 \%$ or higher, as reported in Table 2). This is explained by the hydrophilicity of the CNCs which enabled a good dispersion of the nanoparticles in water but hampered their ability to adsorb and stabilize the oil-water 
interface. On the basis of favorable interactions between water and poly(NIPAM) a preferential affinity of the grafted CNCs is expected with the aqueous phase. Also, according to the Bancroft rule [51] for conventional emulsions and the water contact angle of the grafted CNCs, it was expected that oil-inwater $(\mathrm{O} / \mathrm{W})$ emulsions were formed. This is in fact what was observed, i.e., $\mathrm{O} / \mathrm{W}$ emulsions were obtained in all cases as confirmed by the drop tests.

After 30 minutes, emulsions homogenized with the largest concentration of grafted CNCs $(0.5 \%)$ produced the largest volume fraction of emulsified phase as indicated by Table 2. At lower CNC concentrations, the volume fraction of emulsified phase decreased, as expected due to coalescence of larger oil droplets formed (see Figure 1). Low concentrations of grafted CNCs $(0.05$ and $0.11 \%)$ emulsified the most oil at 94 and $95 \%$, respectively. This was expected due to lower concentrations of nanoparticles being available to stabilize oil-water interfaces leading to larger droplet sizes (discussed later). Higher concentrations yielded smaller droplets, thus a decreased total volume of oil emulsified. After 4 days at ambient conditions, Pickering emulsions prepared with $0.25 \%$ grafted CNCs showed the highest stability index at $94 \%$. However, when the concentration of grafted CNCs was increased to 0.5 $\%$, a lower stability index was noted at $83 \%$. Most likely, an excess of grafted CNCs was present at the oil-water interface and in the continuous (aqueous) phase which was later confirmed in Table 3. Given the tendency of the grafted CNCs to aggregate (discussed in later sections) and the favorable intermolecular interactions between adjacent poly(NIPAM) brushes in hydrophobic environments, this could have assisted the desorption of grafted CNCs from the oil-water interface, and thus oil droplet coalescence. Additionally, it has been proposed in studies of ellipsoidal colloids that the compressional stress at interfaces is relaxed by the flipping of non-spherical particles into an upright position, followed by expulsion from the interface [3]. After 4 days, emulsions stored at ambient conditions showed no phase separation and have been stable for more than four months at the time of writing this manuscript.

Table 2. Phase composition and emulsion stability after emulsification of heptane (organic or oil phase, O) in water $(\mathrm{W})$ at a water-to-oil ratio of $1(\mathrm{WOR}=1)$. The systems were equilibrated at room 
temperature. The volume fraction of emulsified, middle phase (E) is reported along with the excess volume fractions separated on the top (oil phase) and in the bottom (aqueous phase).

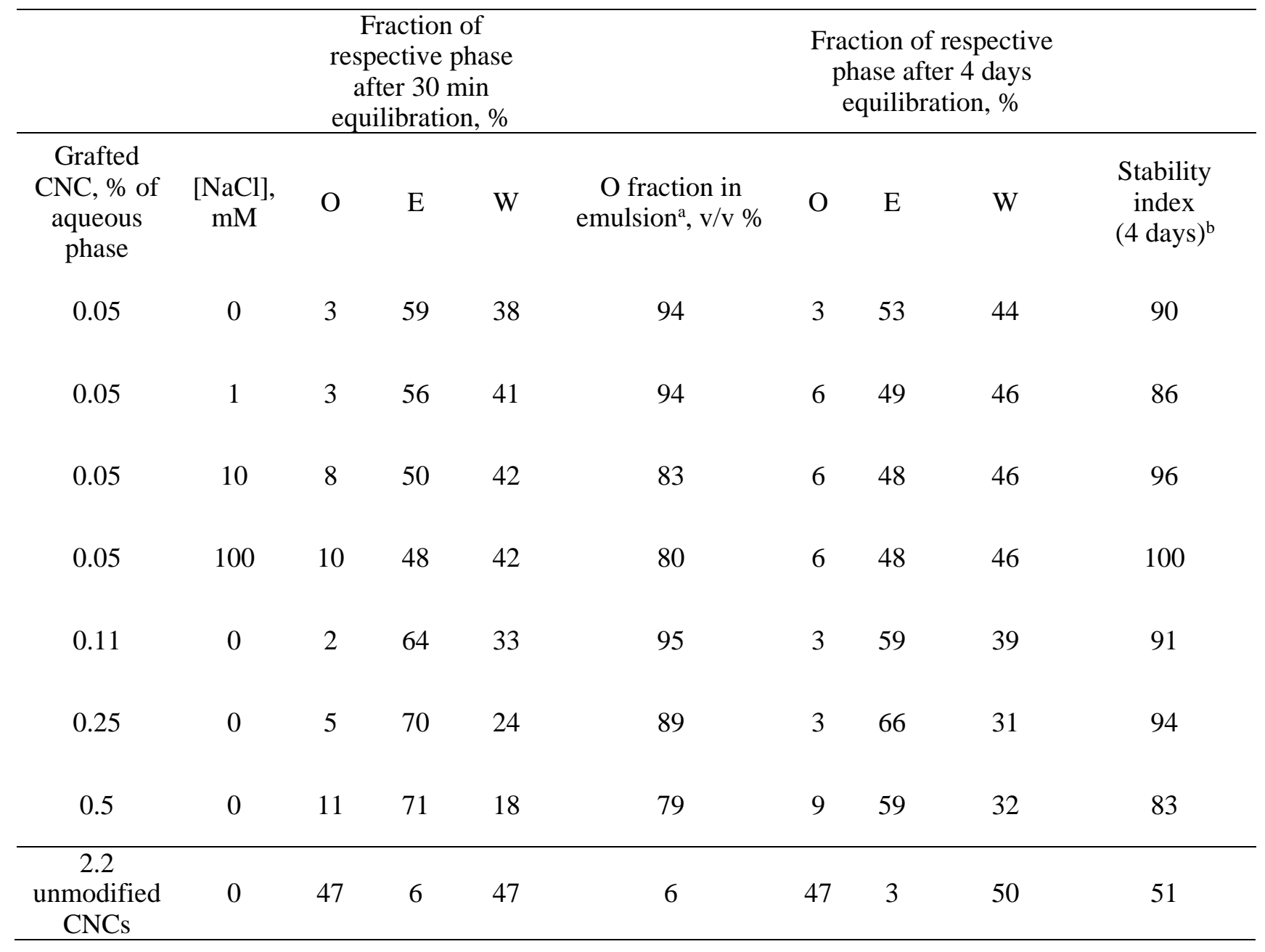

a (Heptane volume fraction in the emulsion)/(total heptane volume $) \times 100$.

$\mathrm{b}$ The stability index was defined as the (Volume fraction of emulsion after 4 days equilibration)/ (Volume fraction emulsion after 30 min equilibration time) $\times 100$. Note that 4 days equilibration time was arbitrarily chosen to measure the stability of the emulsions.

Emulsions prepared with $0.05 \mathrm{wt} \%$ poly(NIPAM)-g-CNCs yielded very large droplets $(>100 \mu \mathrm{m})$, as shown in Figure 1a, compared to those made with higher concentrations, as 0.5 wt \% in Figure 1 b. Larger emulsion droplets were much less stable under low shear by mixing compared to the smaller droplets formed at higher nanoparticle concentrations. Shown in Figure 2(a,b) are results of laser scattering particle size analysis of 0.25 (a) and $0.5 \mathrm{wt} \%$ (b) grafted nanoparticle concentration. In both cases, polydisperse unimodal distributions were obtained with mean particle diameters of about $38 \mu \mathrm{m}$, on number-average basis, which corresponded to optical micrographs (Figure 1b). The highest wt \% 
(0.5) of grafted nanoparticles gave a slightly lower polydispersity than $0.25 \mathrm{wt} \%$. For all cases of emulsions prepared with concentrations lower than $0.25 \mathrm{wt} \%$, the large oil droplets obtained $(>100 \mu \mathrm{m})$ were unstable to shear, and accurate particle size distributions were not obtained.

Effect of ionic strength of the aqueous phase. Emulsion systems with a nanoparticle concentration of $0.05 \mathrm{wt} \%$ were used to study the effect of the ionic strength of the aqueous phase, especially with regard to emulsion stability. Aqueous solutions with 1, 10, and $100 \mathrm{mM} \mathrm{NaCl}$ concentrations were added to aqueous dispersions containing the respective amount of grafted CNCs. After addition of an equivalent amount of heptane (for a WOR=1) and emulsification, it was observed that the ionic strength had a negative effect on the volume fraction of the emulsified phase, according to Table 2 . It is hypothesized that the partial collapse of poly(NIPAM) brushes grafted from CNCs as determined in a previous study [49] made the nanoparticle surfaces less hydrophilic and produced less stable oil droplets. As reported by Saigal et al. [34], low ionic strength gave higher interfacial tension at the oilwater interface, most likely due to lateral repulsive forces of charged nanoparticles. Also, increased ionic strength yielded less electrostatic repulsion between dispersed oil droplets, thus were more likely to coalesce [52]. In addition, the amount of oil emulsified also decreased with ionic strength. However, after a period of 4 days, the emulsions prepared with $100 \mathrm{mM} \mathrm{NaCl}$ showed the most stable emulsified phase.

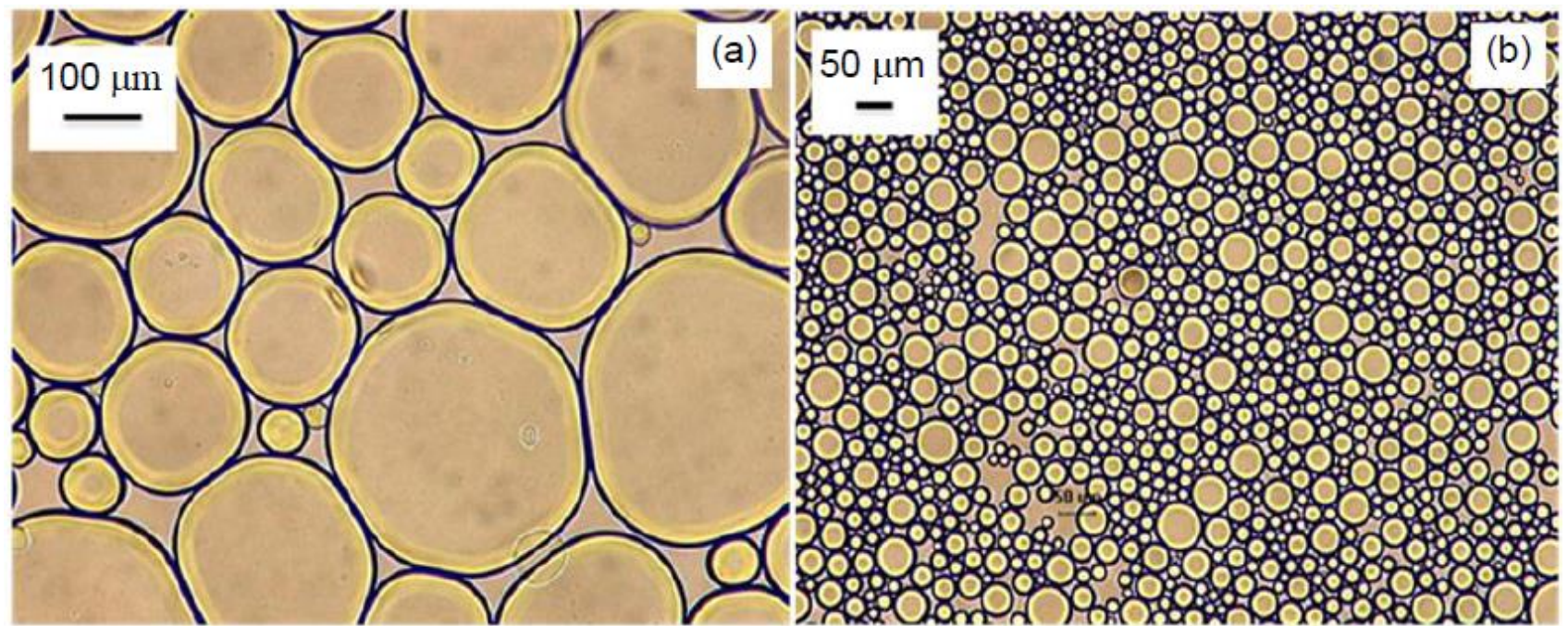

Figure 1. Optical Microscopy Images of emulsions prepared with 0.05 (a) and $0.5 \%$ (b) poly(NIPAM)-g-CNCs using a $\mathrm{WOR}=1$. 

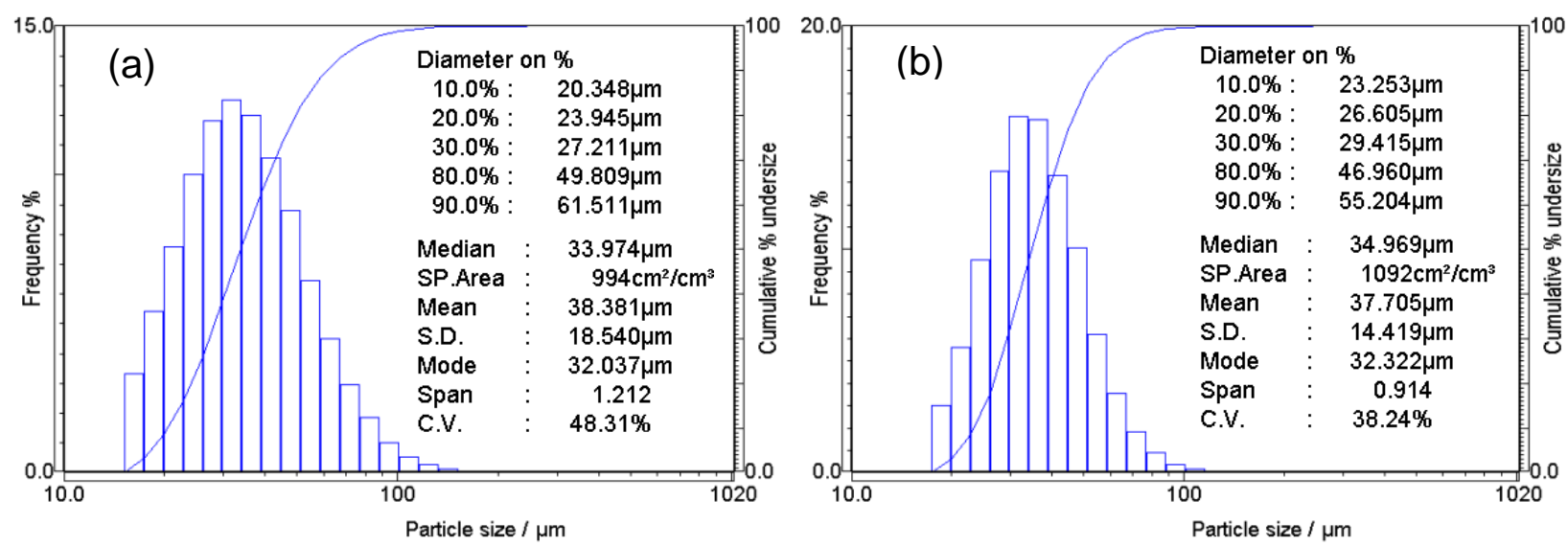

Figure 2. Drop size analysis by laser light scattering of emulsions prepared with 0.25 (a) and $0.5 \%$ (b) poly(NIPAM)-g-CNCs using a WOR=1.

In order to determine the nanoparticle wt \% needed to stabilize Pickering emulsions, samples were extracted from the aqueous phase of formulations without salt added and nanoparticle concentration was calculated gravimetrically (seen in Table 3 ). In the case of $0.05 \mathrm{wt} \%$ formulations, there was not a detectable amount of grafted $\mathrm{CNCs}$ in the excess aqueous phase. However, in cases of higher concentration, significant amounts of grafted CNCs were in the aqueous phase. Thus, it was assumed that the optimum concentration of nanoparticles for these formulations was about $0.25 \mathrm{wt} \%$.

Table 3. Pickering emulsion nanoparticle composition of selected samples (determined gravimetrically).

\begin{tabular}{|c|c|c|c|}
\hline Sample Phase & \multicolumn{3}{|c|}{ wt \% } \\
\hline Original content in aqueous phase & 0.05 & 0.25 & 0.5 \\
\hline Aqueous phase content after emulsification & 0.0 & 0.08 & 0.2 \\
\hline Emulsion phase content after emulsification ${ }^{a}$ & 0.05 & 0.17 & 0.3 \\
\hline
\end{tabular}

Samples of the Pickering emulsions formulated with 0.25 wt $\%$ poly(NIPAM)-g-CNCs were subjected to Freeze-fracture Electron Microscopy (FFEM), in which the emulsion was frozen in propane 
$\left(-189^{\circ} \mathrm{C}\right)$ and fractured in a cryo-chamber, followed by shadowing with $\mathrm{Pt} / \mathrm{C}$ to create a mask so as to be further examined in a Transmission Electron Microscopy (TEM). Individualized poly(NIPAM)-gCNCs were observed on the TEM grids containing freeze-fractured samples. It is possible that they were excess $\mathrm{CNCs}$ or they were broken away from the Pickering emulsions during sample preparation (Figure 3). CNCs dimensions correlated well with those determined previously by AFM [48], however some large aggregates can be noted in Figure 3.

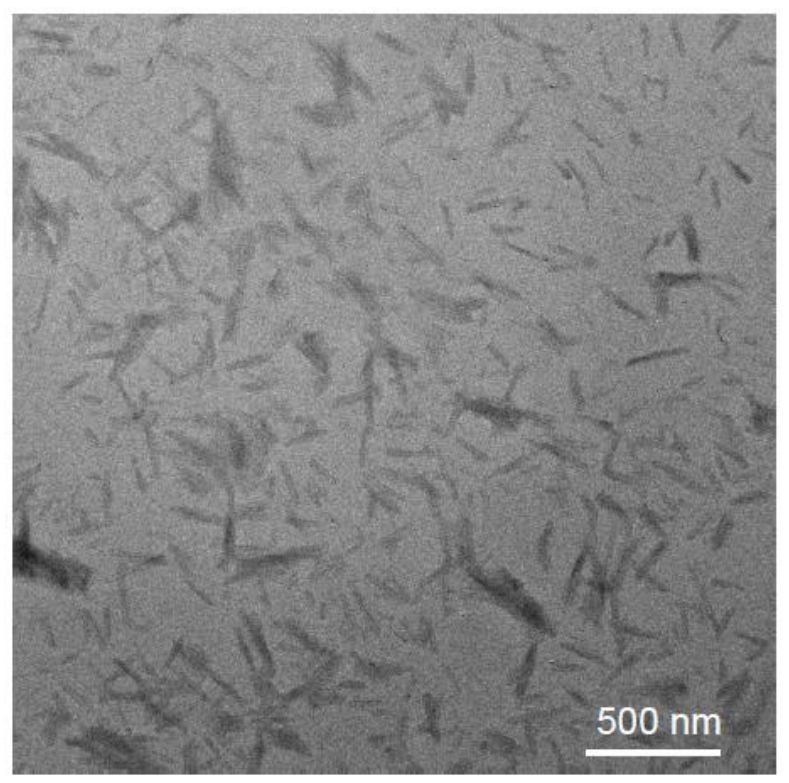

Figure 3. TEM images of poly(NIPAM)-g-CNCs scattered on TEM grids, which were possibly broken away after freeze-fracture of $0.25 \mathrm{wt} \%$ containing Pickering emulsions.

Figure 4 shows TEM images of a freeze-fractured Pickering emulsion droplet stabilized by poly(NIPAM)-g-CNCs at the oil-water interface. The appearance of anisotropically aligned rectangular shaped inclusions suggested that grafted CNCs are aligned at the oil-water interface of Pickering emulsions. It is well known that above a critical solids content $(>1 \%)$, CNCs can form anisotropic assemblies [18]. It was interesting to note that the initial concentrations of grafted nanoparticles in all emulsions prepared was less than $1 \%$, however, due to the formation of three phase systems, the local concentration of grafted nanoparticles in the emulsified phase was expected to be much higher. Interestingly, the size of inclusions visualized $(\sim 500 \mathrm{~nm} \mathrm{x} \sim 2 \mu \mathrm{m})$ when compared to the size of grafted CNCs in Figure 3 suggests large aggregations of grafted CNCs to form microparticles. This finding was in agreement with a study performed by Lin et al. [53], in which larger particles were more strongly 
confined to the oil-water interface than smaller particles, indicating more effective emulsification by micron-sized particles. According to a recent review [3], flocculated dispersions have been reported to be more efficient emulsion stabilizers, and the desorption energy of spherical particles from the interface is directly proportional to the square of the radius, $\varepsilon \sim a^{2}$.

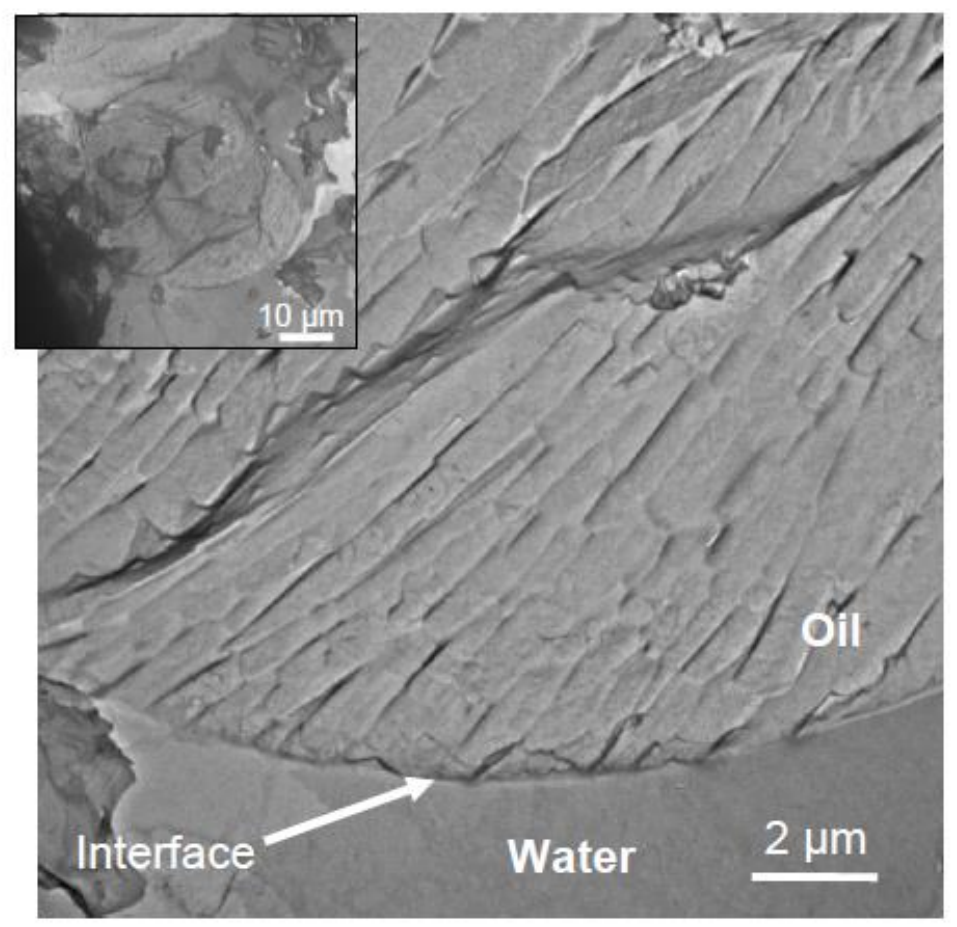

Figure 4. TEM image of the replica oil-water interface of an emulsion droplet after freeze-fracture and shadowed with a 2-3 nm layer of $\mathrm{Pt} / \mathrm{C}$.

Shown in Figure 5 is another emulsion droplet present on the same freeze-fractured sample as previously mentioned viewed by SEM. In this case, SEM showed surface-related features which were not observed in TEM. Figure 5a shows the appearance of fractured crystals at the oil-water interface, which were also aligned. At higher magnification, Figure 6b, the appearance of sheeted layers may be an indication that grafted CNCs not only aggregated laterally with adjacent CNCs, but also aggregated into sheets forming larger structures with lateral dimensions $>1.5 \mu \mathrm{m}$ at the oil-water interface. 


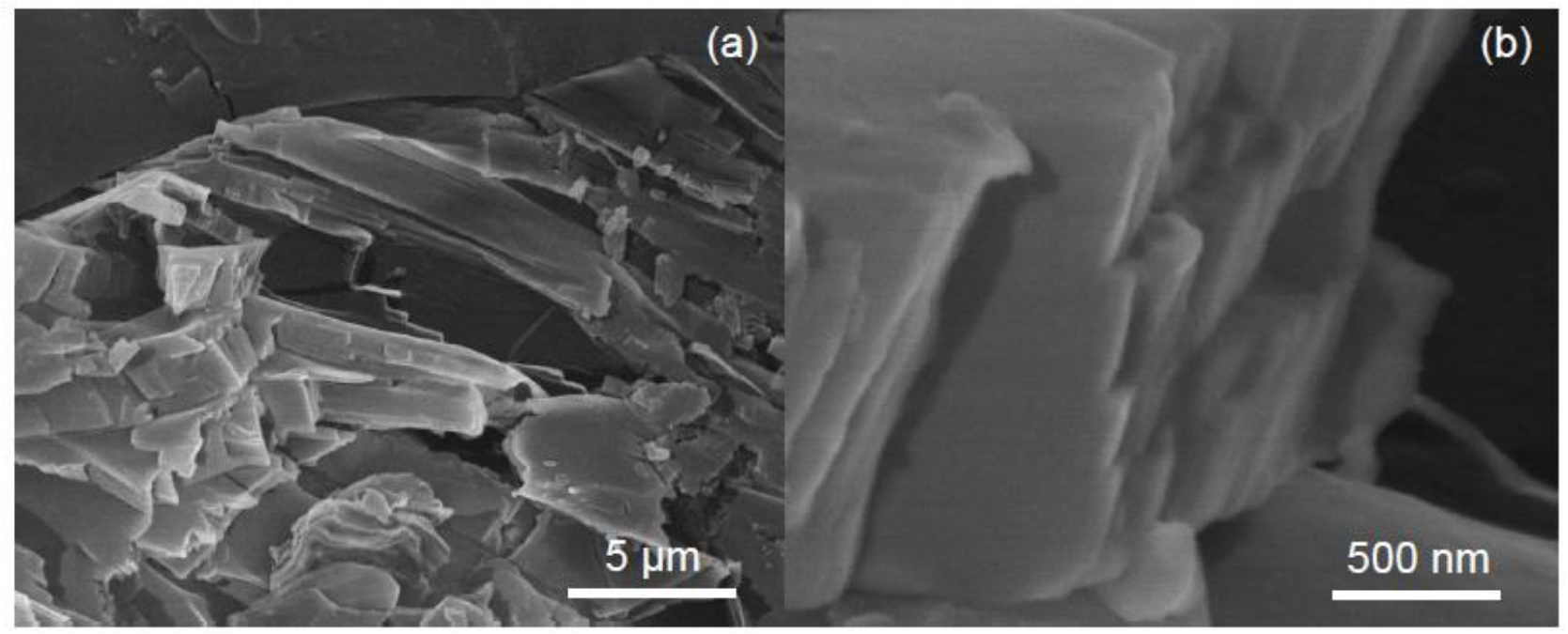

Figure 5. SEM images of fractured poly(NIPAM)-g-CNCs at oil-water interface (a), and layers of broken poly(NIPAM)-g-CNCs after freeze-fracture (b).

Effect of temperature. The stability of Pickering emulsions with nanoparticles carrying thermoresponsive polymer brushes was expected to depend on temperature. Emulsions prepared were stable $>$ 4 months, however after heating above the LCST of poly(NIPAM) the emulsions broke. Pickering emulsions prepared with $0.25 \mathrm{wt} \%$ poly(NIPAM)-g-CNCs were heated above ca. $35^{\circ} \mathrm{C}$ for one minute and as a result emulsions destabilization and phase separation occurred. In addition, the separated, lower aqueous phase became turbid to the naked eye due to grafted nanoparticle aggregation. Thus it is possible that together with particle aggregation, poly(NIPAM)-g-CNCs desorbed from the oil-water interface leading to destabilization of the interface.

To further determine the effect of temperature on Pickering emulsion stability, rheological measurements were performed. Emulsion samples were sheared at a constant rate of $100 \mathrm{~s}^{-1}$ for a temperature ramp of 25 to $45^{\circ} \mathrm{C}$. The viscosity of Pickering emulsions was expected to depend on shear rate [54], however a constant shear rate was chosen to demonstrate temperature effects due to thermo-responsive polymer grafts based on previous work [49]. Shown in Figure 6 are the resulting viscosity versus temperature curves of emulsions prepared from four concentrations of poly(NIPAM)-gCNCs without the addition of salt. The lowest concentration of $0.05 \mathrm{wt} \%$ (Figure 6) showed drastic instability after reaching the LCST at ca. $33{ }^{\circ} \mathrm{C}$ due to emulsions being broken and phase separated. 
When higher concentrations were sheared with temperature, apparent increases in viscosities were observed at the LCST, seen in Figure 6. Interestingly, the peak in viscosity for emulsions prepared with 0.1 wt $\%$ grafted nanoparticles were $1-2{ }^{\circ} \mathrm{C}$ lower than others (shown more clearly in the insert of Figure 6). Also, the viscosity after the LCST was clearly more stable with concentrations at 0.1 and $0.25 \mathrm{wt} \%$, which may be an indication of less significant phase separation. At concentrations of $0.5 \mathrm{wt}$ $\%$ grafted nanoparticles, the initial viscosity was significantly higher $(\sim 10 \mathrm{x})$ than for lower concentrations most likely due to smaller particle sizes. Additionally, a broader transition in increased viscosity was observed at the LCST from $\sim 32.5$ to $36.5^{\circ} \mathrm{C}$, followed by a steady decrease to a similar value as the other emulsion samples. In the case of unmodified CNCs in water, the viscosity only slightly decreased with temperature and did not show thermo-responsiveness, as reported in a previous publication for cellulose nanocrystals [49]. The observed changes in viscosity for all samples were clear indications of emulsions being broken and oil droplet coalescence due to collapse of poly(NIPAM) brushes grafted from CNCs.

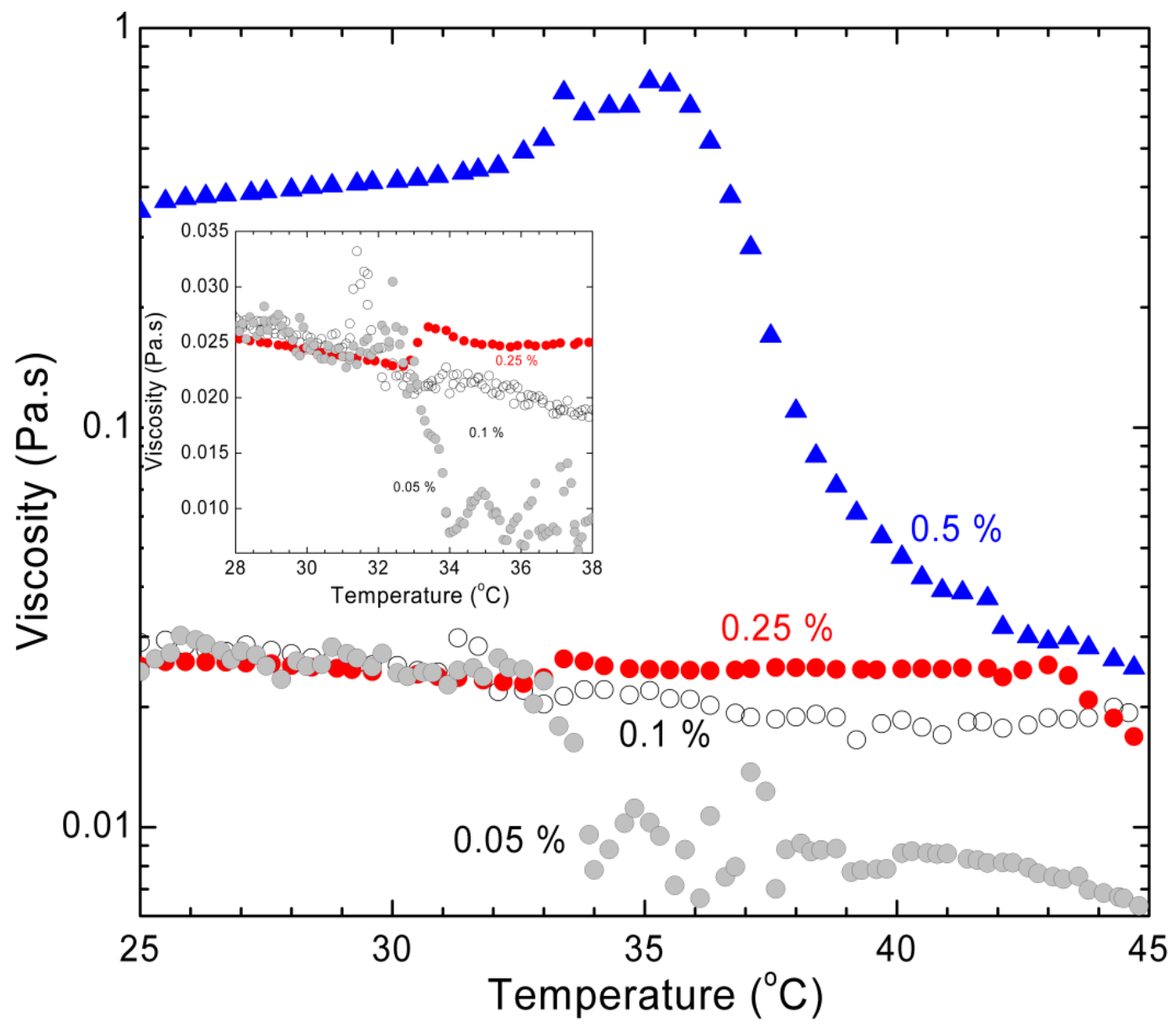


Figure 6. Viscosity $\left(100 \mathrm{~s}^{-1}\right)$ versus temperature for Pickering emulsions prepared with 0.05 (grey circles), 0.1 (open circles), 0.25 (filled circles), and 0.5 (triangles) wt \% poly(NIPAM)-g-CNCs (note semi-log scale). Inset: enhanced view of $0.05,0.1$ and $0.25 \%$ poly(NIPAM)-g-CNCs (linear scale).

The effect of ionic strength on viscosity curves with temperature was also studied for Pickering emulsions prepared with 0.05 wt \% poly(NIPAM)-g-CNCs (see Figure 7). As previously mentioned, emulsions samples prepared with $0.05 \mathrm{wt} \%$ grafted nanoparticles without salt showed instability at $\sim 32$ ${ }^{\circ} \mathrm{C}$, the LCST (Figure 6). At 1 or $100 \mathrm{mM} \mathrm{NaCl}$ concentration in the aqueous phase, viscosity curves were more stable (did not show drastic changes) at around the LCST (Figure 7), most likely explained by decreased steric and electrostatic repulsion between poly(NIPAM)-g-CNCs at the surfaces of oil droplets.

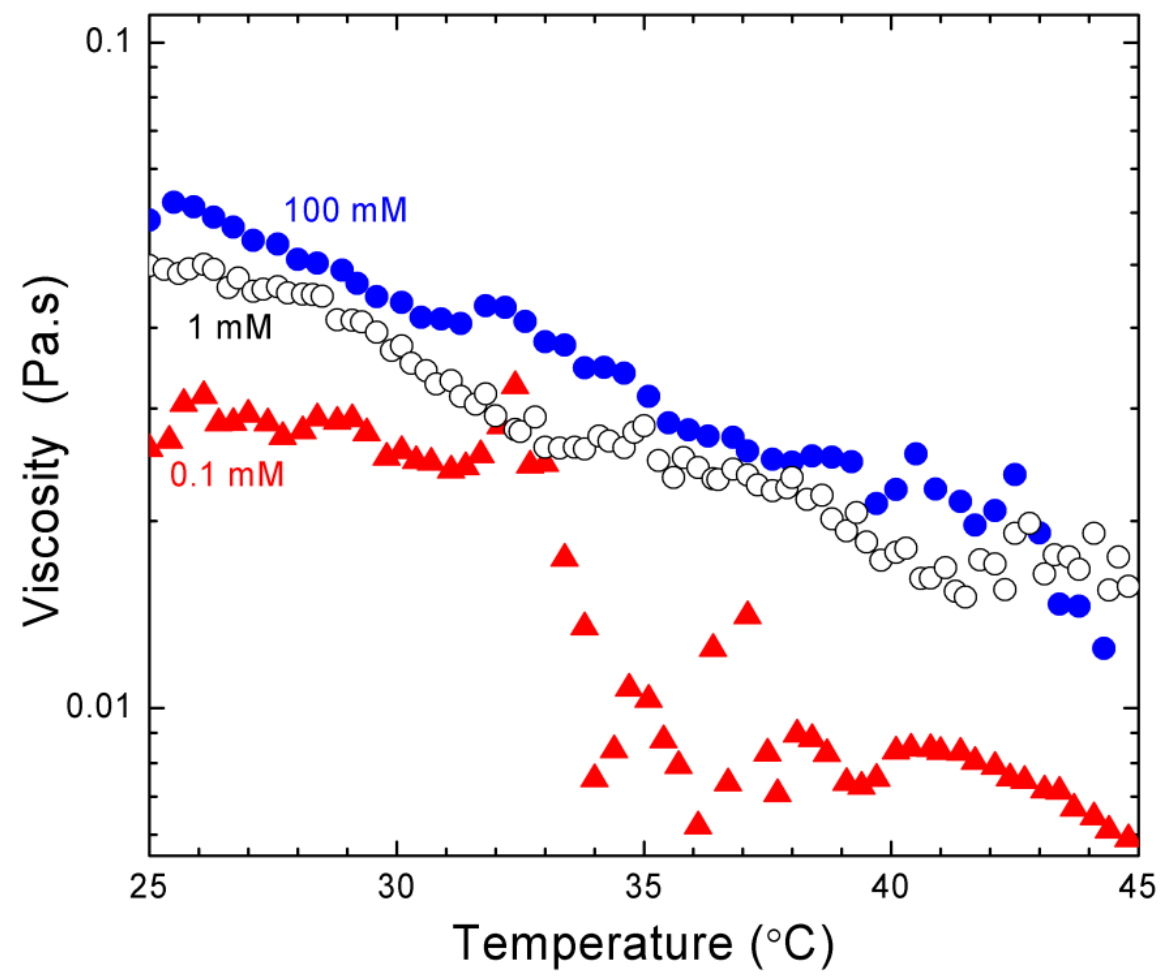

Figure 7. Viscosity $\left(100 \mathrm{~s}^{-1}\right)$ versus temperature for Pickering emulsions prepared with $0.05 \mathrm{wt} \%$ poly(NIPAM)-g-CNCs at 0.1 (triangles), $1 \mathrm{mM}$ (open circles), and $100 \mathrm{mM} \mathrm{NaCl}$ (filled circles) (note $\log$ scale).

\section{CONCLUSIONS}


Thermally responsive heptane-in-water Pickering emulsions were successfully prepared with cellulose nanocrystals grafted with thermo-responsive poly(NIPAM) brushes. Emulsions prepared with 0.05 to 0.5 wt $\%$ poly(NIPAM)-g-CNCs were observed to be stable during the time of observation of four months. Depending on the concentration of grafted nanoparticles, heptane droplets obtained ranged from 30 to $>100 \mu \mathrm{m}$ and showed polydisperse drop size distributions. All emulsions were broken when heated above the LCST of poly(NIPAM) for one minute. This phenomenon was further elucidated via rheological measurements, in which viscosities of emulsion samples increased on approach of the LCST, and smaller drop sizes showed significantly higher viscosities. The ionic strength of emulsions showed a stabilization effect on the viscosity explained by decreased electrostatic and steric repulsion between poly(NIPAM)-g-CNCs at the oil-water interface. The anisotropy of grafted nanoparticles at the oil-water interface was observed via Freeze-fracture TEM. In addition, larger layered sheet assemblies $(>1.5 \mu \mathrm{m})$ were observed as an indication of grafted nanoparticle aggregation during emulsification at the oil-water interface. It is expected that the development of thermally responsive Pickering emulsions utilizing naturally abundant substrates with controllable stability will allow significant advances for biomedical and cosmetic applications.

\section{Acknowledgments}

The authors would like to acknowledge Dr. Rami Al-Khatib for the preparation of freeze-fractured samples, Roberto Garcia for TEM images, and Chuck Mooney for SEM images. This project was supported by the National Research Initiative of the USDA Cooperative State Research, Education and Extension Service, grant number 2007-35504-18290. Also, USDA's NNF program grant number 200738420-17772 and TEKES's LignoCell (Finland) are greatly appreciated.

\section{References}

[1] W. Ramsden, Proc. R. Soc. London, Ser. A 72 (1903) 156.

[2] S.U. Pickering, J. Chem. Soc., Trans. 91 (1907) 2001. 
[3] F. Leal-Calderon, V. Schmitt, Curr. Opin. Colloid Interface Sci. 13 (2008) 217.

[4] S. Abend, N. Bonnke, U. Gutschner, G. Lagaly, Colloid Polym. Sci. 276 (1998) 730.

[5] N. Saleh, T. Phenrat, K. Sirk, B. Dufour, J. Ok, T. Sarbu, K. Matyjaszewski, R.D. Tilton, G.V. Lowry, Nano Lett. 5 (2005) 2489.

[6] N.P. Pardhy, B.M. Budhlall, Langmuir 26 (2010) 13130.

[7] J. Li, H.D.H. Stover, Langmuir 26 (2010) 15554.

[8] J. Li, H.D.H. Stover, Langmuir 27 (2011) 861.

[9] J. Li, A.P. Hitchcock, H.D.H. Stover, Langmuir 26 (2010) 17926.

[10] Y. He, Mater. Lett. 59 (2005) 114.

[11] J.J. Blaker, K.-Y. Lee, X. Li, A. Menner, A. Bismarck, Green Chem. 11 (2009) 1321.

[12] G. Jutz, A. Boeker, J. Mater. Chem. 20 (2010) 4299.

[13] W. Hamad, Can. J. Chem. Eng. 84 (2006) 513.

[14] S. Kamel, eXPRESS Polym. Lett. 1 (2007) 546.

[15] A. Dufresne, Can. J. Chem. 86 (2008) 484.

[16] Y. Habibi, L.A. Lucia, O.J. Rojas, Chem. Rev. 110 (2010) 3479.

[17] X.M. Dong, J.-F. Revol, D.G. Gray, Cellulose 5 (1998) 19.

[18] S. Elazzouzi. Autoorganisation de whiskers de cellulose en suspension dans l'eau ou dans les solvents organiques apolaires. PhD thesis, Joseph Fourier University, Grenoble, 2006.

[19] M. Andresen, L.-S. Johansson, B.S. Tanem, P. Stenius, Cellulose (Dordrecht, Neth.) 13 (2006) 665.

[20] K.P. Oza, S.G. Frank, J. Dispersion Sci. Technol. 7 (1986) 543.

[21] W.R. Thomas, Prog. Food Nutr. Sci. 6 (1982) 341.

[22] H.A. Wege, S. Kim, V.N. Paunov, Q. Zhong, O.D. Velev, Langmuir 24 (2008) 9245.

[23] H. Ougiya, K. Watanabe, Y. Morinaga, F. Yoshinaga, Biosci., Biotechnol., Biochem. 61 (1997) 1541. 
[24] M.J. Cash, A.N. Chan, H.T. Conner, P.J. Cowan, R.A. Gelman, K.M. Lusvardi, S.A. Thompson, F.P. Tise, Derivatized microfibrillar polysaccharides, their formation and use in dispersions. WO $2000047628,2000$.

[25] F. Tournilhac, R. Lorant, Oil-in-water cosmetic emulsion composition containing cellulose fibrils. EP 1057477, 2000.

[26] M. Andresen, P. Stenius, J. Dispersion Sci. Technol. 28 (2007) 837.

[27] A. Lif, P. Stenstad, K. Syverud, M. Nyden, K. Holmberg, J. Colloid Interface Sci. 352 (2010) 585.

[28] K. Xhanari, K. Syverud, G. Chinga-Carrasco, K. Paso, P. Stenius, J. Colloid Interface Sci. 356 (2011) 58 .

[29] K. Xhanari, K. Syverud, P. Stenius, J. Dispersion Sci. Technol. 32 (2011) 447.

[30] I. Kalashnikova, H. Bizot, B. Cathala, I. Capron, Langmuir 27 (2011) 7471.

[31] M.R. Mauricio, G.M. Carvalho, E. Radovanovic, E.C. Muniz, A.F. Rubira, Mater. Sci. Eng., C 29 (2009) 594.

[32] W. Wang, L. Chen, J. Appl. Polym. Sci. 104 (2007) 1482.

[33] J. Yin, Z. Ge, H. Liu, S. Liu, J. Polym. Sci., Part A: Polym. Chem. 47 (2009) 2608.

[34] T. Saigal, H. Dong, K. Matyjaszewski, R.D. Tilton, Langmuir 26 (2010) 15200.

[35] B.P. Binks, R. Murakami, S.P. Armes, S. Fujii, Angew. Chem., Int. Ed. 44 (2005) 4795.

[36] H.G. Schild, Prog. Polym. Sci. 17 (1992) 163.

[37] M. Hamidi, A. Azadi, P. Rafiei, Adv. Drug Delivery Rev. 60 (2008) 1638.

[38] M. Kawaguchi, Y.-i. Hirose, T. Kato, Langmuir 12 (1996) 3523.

[39] J. Zhang, R. Pelton, Langmuir 12 (1996) 2611.

[40] M. Kawaguchi, W. Saito, T. Kato, Macromolecules 27 (1994) 5882.

[41] S. Tsuji, H. Kawaguchi, e-Polym. (2005) No pp given.

[42] K. Chan, R. Pelton, J. Zhang, Langmuir 15 (1999) 4018.

[43] T. Ngai, H. Auweter, S.H. Behrens, Macromolecules 39 (2006) 8171.

[44] T. Ngai, S.H. Behrens, H. Auweter, Chem. Commun. (Cambridge, U. K.) (2005) 331. 
[45] D.B. Lawrence, T. Cai, Z. Hu, M. Marquez, A.D. Dinsmore, Langmuir 23 (2007) 395.

[46] T. Suzuki, C. Morishita, M. Kawaguchi, J. Dispersion Sci. Technol. 31 (2010) 1479.

[47] S. Tsuji, H. Kawaguchi, Langmuir 24 (2008) 3300.

[48] J.O. Zoppe, Y. Habibi, O.J. Rojas, R.A. Venditti, L.-S. Johansson, K. Efimenko, M. Osterberg, J. Laine, Biomacromolecules 11 (2010) 2683.

[49] J.O. Zoppe, M. Osterberg, R.A. Venditti, J. Laine, O.J. Rojas, Biomacromolecules 12 (2011) 2788.

[50] D.R. Lide, CRC Handbook of Chemistry and Physics. CRC Press LLC, Boca Raton, 2003.

[51] W.D. Bancroft, J. Phys. Chem. 17 (1913) 501.

[52] N.P. Ashby, B.P. Binks, Phys. Chem. Chem. Phys. 2 (2000) 5640.

[53] Y. Lin, A. Boeker, H. Skaff, D. Cookson, A.D. Dinsmore, T. Emrick, T.P. Russell, Langmuir 21 (2005) 191.

[54] R. Aveyard, B.P. Binks, J.H. Clint, Adv. Colloid Interface Sci. 100-102 (2003) 503. 